\title{
Tendência secular da doença diarréica na infância na cidade de São Paulo (1984- 1996)*
}

\section{Secular trends in child diarrhea in S. Paulo city, Brazil (1984-1996)}

\author{
Maria Helena D'Aquino Benicio e Carlos Augusto Monteiro \\ Departamento de Nutrição da Faculdade de Saúde Pública da Universidade de São Paulo. São Paulo, \\ SP, Brasil
}

\section{Descritores}

Diarréia infantil, epidemiologia\#.

Levantamentos epidemiológicos".

Fatores socioeconômicos.

Aleitamento materno. Hospitalização. Séries de tempo. Estudos transversais.

\section{Resumo}

\section{Objetivo}

Estimar a prevalência e a distribuição social da doença diarréica na infância, estabelecer a tendência secular dessa enfermidade e analisar sua determinação, através dos dados coletados por dois inquéritos domiciliares realizados na cidade de São Paulo, SP, em 1984/85 e 1995/96.

\section{Métodos}

Os inquéritos estudaram amostras probabilísticas da população residente na cidade com idades entre zero e 59 meses (1.016 em 1984/85 e 1.280 em 1995/ 96). Nos dois inquéritos foram estimadas a prevalência instantânea da diarréia (proporção de crianças com três ou mais evacuações líquidas no dia da entrevista domiciliar) e a incidência anual de internações hospitalares pela doença. Esses dois indicadores foram calculados a partir de entrevistas domiciliares feitas por médicos pediatras e respondidas pelas mães das crianças. Nos dois inquéritos, as entrevistas foram distribuídas ao longo de um período de cerca de 12 meses, de modo a garantir uma varredura uniforme das várias áreas da cidade ao longo das quatro estações do ano. O estudo da distribuição social da doença diarréica levou em conta tercis da renda familiar per capita em cada um dos inquéritos. A estratégia analítica para estudar os determinantes da evolução da prevalência da doença na população empregou modelos hierárquicos de causalidade, análises multivariadas de regressão e procedimentos análogos aos utilizados para calcular riscos atribuíveis populacionais.

\section{Resultados/Conclusões}

Houve entre os inquéritos reduções expressivas na prevalência instantânea da diarréia (de $1,70 \%$ para $0,90 \%$ ) e na incidência anual de hospitalizações pela doença (de 2,21 para 0,79 internações por 100 crianças-ano). O declínio desses indicadores foi mais intenso no terço mais pobre da população, o que contribuiu para reduzir a desigualdade social quanto à ocorrência da doença. Melhorias no poder aquisitivo das famílias e na cobertura da rede pública de abastecimento de água justificariam parte considerável do declínio na prevalência da diarréia, havendo ainda indicação de que, entre crianças menores de dois anos, esse declínio possa ter sido favorecido por um aumento discreto na frequiência da amamentação.
Correspondência para/Correspondence to: Maria Helena D'Aquino Benicio Av. Dr. Arnaldo, 715

01246-904 São Paulo, SP, Brasil

E-mail: benicio@usp.br
* Trabalho desenvolvido no Departamento de Nutrição da Faculdade de Saúde Pública da Universidade de São Paulo e no Núcleo de Pesquisas Epidemiológicas em Nutrição e Saúde da Universidade de São Paulo e baseado em pesquisas de campo financiadas pela Financiadora de Estudos e Projetos - Finep (Convênios 41.83.0698.00 e 66.96.0193.00) e pela Fundação de Amparo à Pesquisa do Estado de São Paulo - Fapesp (Processos 84/2463-3 e 94/3493-5). 


\section{Keywords}

Diarrhea, infantile, epidemiology $y^{\#}$. Health surveys". Socioeconomic factors. Breast-feeding.

Hospitalization. Time series. Crosssectional studies.

\begin{abstract}
Objective

Data from two consecutive household surveys undertaken in mid-80s and mid-90s allow to characterize and analyse secular trends in infant and child diarrhea in the city of S. Paulo, Brazil.

Methods

The two surveys included random population samples aged from zero to 59 months of age (1,016 in the period of 1984-85 and 1,280 in 1995-96). In both surveys the instant prevalence of diarrhea (proportion of examined individuals reporting three or more episodes of liquid stools in 24 hours) and the annual incidence of hospitalizations due to the disease were calculated. These two indicators were estimated from household interviews conducted by pediatricians with the children's mothers. In both surveys the interviews were distributed throughout a period of almost 12 months to assure a uniform coverage of the various areas of the city during the four seasons. For each survey, the study of the social distribution of the disease took into account tertiles of the per capita family income. For the study of the determinants of secular trends, hierarchical causal models, multivariate regression analyses and calculations analogous to the ones used to assess population attributable risks were applied.

Results/Conclusions

In the time span from the first to the second survey, there was substantial reduction in both the prevalence of diarrhea (from $1.70 \%$ to $0.90 \%$ ) and the hospitalizations due to the disease (from 2.21 to 0.79 hospitalizations per 100 children-year). A more significant reduction was observed among the third poorest families, narrowing the social gradient relative to the disease. An increase in family income and improvement in water supply could substantially explain part of the decline in the disease and, for children under two years of age, a discrete increase in breast-feeding may have also played a positive role.
\end{abstract}

\section{INTRO DU ÇÃOO}

A doença diarréica em crianças é um dos maiores problemas de saúde enfrentados pelos países em desenvolvimento, constituindo, nessas sociedades, uma das principais causas de mortalidade precoce, um determinante crucial do retardo do crescimento na infância e uma das razões mais freqüentes para procura de serviços de saúde. ${ }^{1,4,12}$

Determinada sobretudo pelas precárias condições do saneamento do meio, a diarréia infantil em países em desenvolvimento alcança incidências que, por vezes, ultrapassam dez episódios por criança por ano. ${ }^{4,6}$ Análises da tendência secular da diarréia infantil nesses países têm indicado declínios da mortalidade, mas não da incidência da doença. ${ }^{4}$

Inquérito domiciliar probabilístico realizado no Brasil em 1989 estimou em 10,5\% a prevalência de crianças menores de cinco anos com diarréia nas duas últimas semanas, oscilando essa prevalência entre $5,9 \%$ na Região Sul e $15,4 \%$ na Região Nordeste. ${ }^{3}$ Inquéritos semelhantes realizados em vários estados da Região Nordeste entre 1989 e 1992 evidenciaram prevalência instantânea da diarréia (crianças com diarréia no dia da entrevista) variando entre 4,5\% (Bahia e Rio Grande do Norte) e $9,5 \%$ (Alagoas e Ceará). ${ }^{17}$
Como em outros países em desenvolvimento, a mortalidade infantil por doença diarréica vem declinando no Brasil. . $^{15,18,20}$ Não há muitas informações, entretanto, sobre a tendência secular da morbidade por diarréia em nosso meio. Inquéritos domiciliares probabilísticos realizados na cidade de São Paulo em meados das décadas de 70 e 80 evidenciaram, entre outros aspectos, redução na ocorrência da diarréia infantil e expansão substancial da cobertura da rede pública de abastecimento de água da cidade. ${ }^{2,14}$ Um novo inquérito domiciliar, realizado em São Paulo em 1994/95, permite que se volte a examinar a frequiência, a distribuição social e a tendência secular da diarréia infantil na cidade.

\section{MÉTODOS}

\section{Amostragem}

A frequiência e a distribuição social da doença diarréica infantil em São Paulo foram calculadas com base em inquérito realizado pelo Núcleo de Pesquisas Epidemiológicas em Nutrição e Saúde da Universidade de São Paulo (Nupens/USP), entre setembro de 1995 e agosto de 1996, em uma amostra probabilística de 4.560 domicílios da cidade de São Paulo ("Saúde e nutrição das crianças de São Paulo II"). O processo de amostragem deste inquérito encontra-se detalhado alhures. ${ }^{16}$ Tratouse de uma amostragem estratificada em múltiplas etapas, envolvendo o sorteio de setores censitários, con- 
glomerados de domicílios e domicílios individuais. A visita aos 4.560 domicílios sorteados identificou um total de 1.390 crianças menores de cinco anos. Dessas, 54 $(3,9 \%)$ deixaram de ser estudadas por não serem encontradas no domicílio após um número mínimo de três visitas (sendo pelo menos uma em sábados ou domingos) ou por não concordância dos pais em participar do estudo; outras $56(4,0 \%)$ foram estudadas apenas parcialmente porque mudaram de residência antes que o estudo fosse completado. Assim, foram integralmente estudadas 1.280 crianças com idades entre zero e 59 meses de idade. A cada uma dessas crianças foi associado um fator que representa seu peso na amostra (peso amostral). Esse fator é dado pelo inverso da fração amostral do domicílio onde vive a criança (quantos domicílios da cidade estão sendo representados por aquele domicílio) multiplicado pelo inverso da taxa de sucesso da pesquisa no setor onde se encontra o domicílio. Com a aplicação desses pesos, o conjunto das 1.280 crianças estudadas representa adequadamente o conjunto das crianças menores de cinco anos que vivia na cidade de São Paulo em 1995/96.

A evolução recente da doença diarréica será identificada comparando-se os resultados do inquérito de 1995/96 com resultados de inquérito anterior realizado pelo Nupens/USP, entre abril de 1984 e junho de 1985, em uma amostra probabilística de 3.378 domicílios da cidade de São Paulo, da qual resultou o estudo de 1.016 crianças menores de cinco anos de idade ("Saúde e nutrição das crianças de São Paulo I"). O processo de amostragem do inquérito de 1984/85, descrito em detalhe em outra publicação, ${ }^{13}$ é semelhante ao descrito para o inquérito de 1995/96.

\section{Coleta de dados e indicadores da freqüência da doença diarréica}

Procedimentos idênticos para a coleta de dados foram empregados pelos inquéritos realizados na cidade de São Paulo em 1984/85 e 1995/96. As visitas aos domicílios sorteados foram distribuídas ao longo de 14 meses no primeiro inquérito (abril de 1984 a junho de 1985) e ao longo de 12 meses no segundo inquérito (setembro de 1995 a setembro de 1996), de modo a garantir uma varredura uniforme das várias regiões da cidade ao longo das quatro estações do ano. A uma equipe de sociólogos, experientes em inquéritos domiciliares, coube identificar os domicílios onde residiam crianças menores de cinco anos, proceder à caracterização socioeconômica das famílias dessas crianças e obter autorização para o exame clínico das mesmas. O exame clínico e a anamnese das crianças (incluindo questões sobre antecedentes de diarréia) foram realizados nos próprios domicílios, imediatamente após a visita inicial, por uma segunda equipe composta por médicos pediatras devidamente treina- dos. A esses médicos coube elaborar relatórios médicos e correspondentes diagnósticos que, posteriormente, eram revistos pelo supervisor clínico do estudo (também médico pediatra). Nos dois inquéritos, adotando-se definição da Organização Mundial da Saúde, a ocorrência da doença diarréica foi identificada pela presença de três ou mais evacuações líquidas no dia. No caso particular de crianças menores de seis meses, acresceu-se ao critério anterior o relato obrigatório de mudança nítida no padrão de evacuação da criança. ${ }^{21}$

Os seguintes indicadores da frequiência de doença diarréica foram empregados no presente estudo:

- prevalência no dia (ou instantânea) $\rightarrow$ percentual de crianças com diarréia no dia da entrevista;

- prevalência nas duas últimas semanas $\rightarrow$ percentual de crianças cujas mães relatam ocorrência de diarréia nas duas semanas anteriores à entrevista;

- incidência anual de hospitalizações $\rightarrow$ número de internações hospitalares devidas à diarréia por 100 crianças-ano.

Adicionalmente, para fins de comparação com outros inquéritos, estimou-se a incidência anual de episódios de diarréia. Tal estimativa foi calculada dividindo-se a prevalência da diarréia no dia pelo número de dias estimado para cada episódio de diarréia e, a seguir, multiplicando o resultado obtido pelo número de dias no ano. O número de dias de cada episódio de diarréia foi estimado a partir da duração média do episódio mais recente da doença ocorrido no mês anterior à visita médica: 4,5 dias em 1984/85 e 3,0 dias em 1995/96.

\section{Procedimentos analíticos}

A distribuição social da doença diarréica foi estabelecida a partir de estratificação da amostra estudada no inquérito de 1995/96 segundo categorias da renda familiar e da escolaridade materna. As categorias de renda foram expressas com base na renda familiar per capita em unidades de salário mínimo de outubro de 1996 (0-0,5, 0,5-1,0, 1,0-2,0 e 2,0 ou mais saláriosmínimos per capita), adotando-se como deflator o Índice Nacional de Preços ao Consumidor - INPC acumulado, calculado pelo IBGE. As categorias de escolaridade consideraram os anos de estudo concluídos pela mãe da criança (0-3, 4-7, 8-10 e 11 ou mais anos).

A tendência secular da doença diarréica foi determinada pela comparação da freqüência da enfermidade nos inquéritos de 1984/85 e de 1995/96. Visando apreender o comportamento de desigualdades sociais, a comparação foi feita para a população como um todo e para três estratos, os quais correspondem ao terço mais pobre das crianças do município (o primeiro tercil da renda familiar per capita - RFPC - em cada inquérito), o ter- 
ço intermediário (o tercil intermediário da RFPC em cada inquérito) e o terço das crianças com melhor poder aquisitivo (o tercil superior da RFPC em cada inquérito).

O significado estatístico das diferenças sociais na distribuição da doença diarréica e da evolução temporal da enfermidade foi avaliado mediante a utilização de testes baseados na distribuição do qui quadrado para identificação de heterogeneidade ou de tendência linear. ${ }^{11}$ Os procedimentos analíticos empregados para se investigar as causas subjacentes às variações temporais na freqüência da diarréia infantil na cidade de São Paulo serão explicitados na seção de resultados.

\section{RESULTADOS}

\section{A situação da doença diarréica em 1995/96}

Freqüências relativamente baixas de crianças com diarréia são encontradas na cidade: menos de $5 \%$ no período correspondente às duas semanas anteriores à entrevista e menos de $1 \%$ no dia da entrevista. A partir do mesmo inquérito, estima-se que, ao longo do período de um ano, cada criança da cidade terá, em média, um episódio de diarréia e que, também em média, para cada 100 crianças haverá menos de uma internação hospitalar por diarréia (Tabela 1).

Tabela 1 - Indicadores da freqüência de doença diarréica. Crianças entre zero e 59 meses de idade da cidade de São Paulo, SP, 1995/96.

\begin{tabular}{lc}
\hline \multicolumn{1}{c}{ Indicadores de diarréia } & $(\mathrm{n}=1.277)$ \\
\hline Prevalência no dia (\%) & 0,90 \\
Prevalência nas duas últimas semanas (\%) & 4,70 \\
Incidência anual de episódios & \\
(Episódios por criança-ano) & 1,04 \\
\hline
\end{tabular}

A distribuição da prevalência da doença diarréica (no dia e nas duas últimas semanas) segundo sexo, faixa etária, estação do ano e estrato social é apresentada na Tabela 2. Merece destaque a ocorrência mais freqüente de diarréia observada entre filhos de mães com pouca ou nenhuma escolaridade e entre crianças que vivem em famílias de baixa renda. No caso específico do indicador de prevalência relativo às duas últimas semanas, fica também evidente a tendência de maior ocorrência de diarréia entre os 6 e os 23 meses de idade e nas estações da primavera e do verão.

\section{Tendência secular da doença diarréica}

A comparação entre os inquéritos realizados na cidade de São Paulo em 1984/85 e em 1995/96 indica declínio de quase $50 \%$ na prevalência instantânea da diarréia e redução ainda mais intensa (quase dois terços) na incidência anual de hospitalizações pela doença (Tabela 3). No mesmo período, estima-se que a incidência anual de episódios de diarréia tenha declinado de 1,35 para 1,09 episódio por criança-ano. A inquirição sobre a presença de diarréia nas duas últimas semanas, observada apenas no inquérito mais recente, não permitiu o exame da evolução desse indicador.

Declínios intensos na prevalência instantânea da diarréia e na incidência anual de hospitalizações, próximos a 70\%, são observados para o terço mais pobre das crianças de São Paulo. Nos outros dois terços da população infantil da cidade, o declínio dos indicadores é menos intenso ou mesmo inexiste. Como resultado, a "desigualdade social" quanto à ocorrência da diarréia tende a diminuir entre os inquéritos (Tabela 4).

Tabela 2 - Prevalência de diarréia segundo sexo, idade, estação do ano e estrato social. Crianças entre zero e 59 meses de idade da cidade de São Paulo, SP, 1995/96.

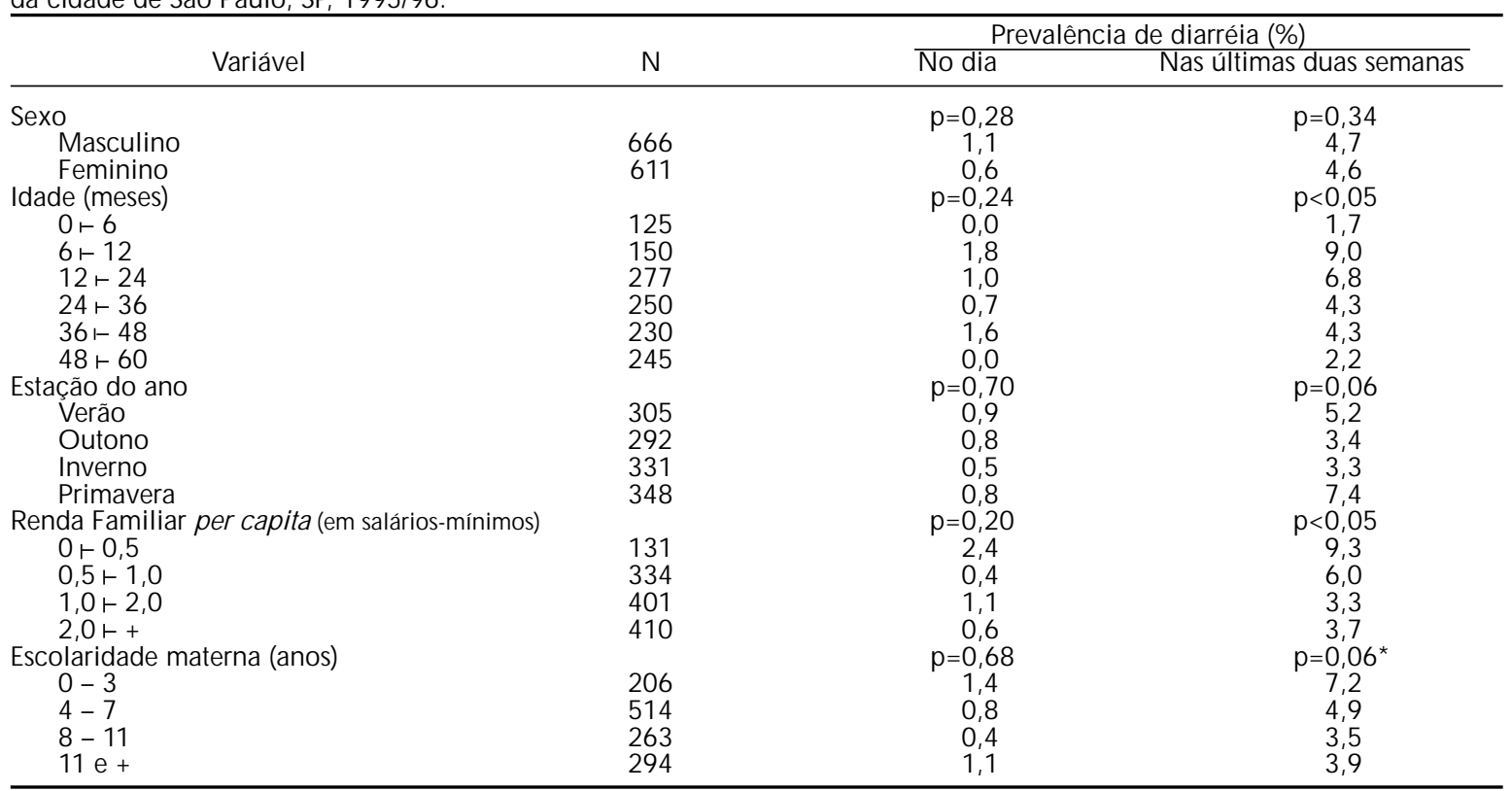

*Tendência linear. 
Tabela 3 - Evolução de indicadores da freqüência de doença diarréica. Crianças entre zero e 59 meses de idade da cidade de São Paulo, SP, 1984/85 e 1995/96.

\begin{tabular}{lccc}
\hline \multicolumn{1}{c}{ Indicadores de diarréia } & $\begin{array}{c}1984 / 85 \\
(\mathrm{n}=1.016)\end{array}$ & $\begin{array}{c}1995 / 96 \\
(\mathrm{n}=1.277)\end{array}$ & $\mathrm{p}$ \\
\hline Prevalência no dia (\%) & 1,70 & 0,90 & 0,07 \\
Incidência anual de hospitalizações (hospitalizações por 100 crianças-ano) & 2,21 & 0,79 & $<0,01$ \\
\hline
\end{tabular}

Tabela 4 - Evolução de indicadores da freqüência da doença diarréica segundo tercis da renda familiar per capita. Crianças entre zero e 59 meses de idade da cidade de São Paulo, SP, 1984/85 e 1995/96.

\begin{tabular}{|c|c|c|c|}
\hline Indicadores / tercis & $\begin{array}{l}1984 / 85 \\
n=1.016)\end{array}$ & $\begin{array}{c}1995 / 96 \\
(n=1.277)\end{array}$ & $\mathrm{p}$ \\
\hline \multirow{2}{*}{$\begin{array}{l}\text { Prevalência no dia (\%) } \\
\text { 33,3\% mais pobres } \\
33,3 \% \text { intermediários } \\
33,3 \% \text { mais ricos } \\
\text { Incidência anual de hospitalizações (hospitalizações por } 100 \text { crianças-ano) } \\
\text { 33,3\% mais pobres } \\
\text { 33,3\% intermediários } \\
\text { 33,3\% mais ricos }\end{array}$} & $\begin{array}{r}2,9 \\
1,5 \\
0,6\end{array}$ & $\begin{array}{l}0,9 \\
1,0 \\
0,7\end{array}$ & $\begin{array}{r}<0,05 \\
0,53 \\
0,90\end{array}$ \\
\hline & $\begin{array}{l}2,4 \\
1,8 \\
2,4\end{array}$ & $\begin{array}{l}0,6 \\
0,7 \\
1,1\end{array}$ & $\begin{array}{c}<0,05^{*} \\
0,15^{*} \\
0,20\end{array}$ \\
\hline
\end{tabular}

*Teste exato de Fisher.

\section{Causas do declínio da doença diarréica}

O estudo das causas responsáveis pela redução da ocorrência de diarréia no período estudado requer a adoção de um modelo teórico que estabeleça as relações hierárquicas entre os fatores que potencialmente contribuem para a determinação da enfermidade. Segundo o modelo adotado neste estudo, a ocorrência da doença diarréia dependeria do grau de exposição a enteropatógenos e da suscetibilidade do organismo infantil. Esses determinantes - os mais proximais - seriam condicionados essencialmente pelo acesso à água tratada e ao saneamento ambiental e pelo estado nutricional da criança. Nos dois primeiros anos de vida, também são muito importantes as práticas de alimentação infantil, em particular a prática do aleitamento materno. Água, saneamento, nutrição e práticas alimentares (determinantes intermediários da diarréia), por sua vez, estariam condicionados pela renda familiar e pela capacidade da família em alocar de forma racional os recursos disponíveis - capacidade que poderia ser indicada pela escolaridade materna. Renda familiar e escolaridade materna seriam, assim, determinantes distais da doença. Por fim, os determinantes distais e todos os fatores que os sucedem no modelo seriam condicionados pela classe social a que a família pertença.

Para identificar, dentre os componentes do modelo de determinação, aqueles que, no período de interesse, se apresentavam efetivamente associados à ocorrência da doença diarréica $(\mathrm{p}<0,10)$, procedeu-se ao acoplamento dos bancos de dados dos inquéritos de 1984/85 e de 1995/96. Sobre essa base combinada de dados, foram realizadas sucessivas análises multivariadas de regressão logística que buscaram, nos diferentes níveis hierárquicos do modelo, atendendo às especificidades do modelo hierárquico, ${ }^{19}$ as variáveis estatisticamente associadas ao risco da do- ença diarréica. Para cada categoria dessas variáveis categoria de base (crianças não expostas) e categoria ou categorias de risco (crianças expostas) - estimouse o risco relativo (nesse caso, "odds ratio") de ocorrência da doença diarréica. A partir desses riscos e das variações temporais (1984/85-1995/96) na frequiência de crianças expostas e não expostas, calculou-se o impacto sobre a evolução da prevalência da doença diarréica que poderia ser atribuído a cada variável. Esse cálculo, análogo ao cálculo do risco atribuível populacional, ${ }^{11}$ é feito contrastando-se os resultados obtidos ao se ponderar os riscos relativos da doença diarréica segundo a proporção de expostos e não expostos no primeiro e no segundo inquérito.

Considerando a disponibilidade de informações nos dois inquéritos e o delineamento transversal dos mesmos, que não permite aferir o sentido da associação entre o estado nutricional infantil e a ocorrência da doença diarréica, as análises conduzidas restringiramse a examinar o papel dos determinantes distais da doença diarréica (renda familiar e escolaridade materna) e o papel de determinantes intermediários para os quais se contava com informações: acesso aos serviços públicos de abastecimento de água e de coleta de esgoto e a prática de aleitamento materno, este último considerado apenas para as crianças menores de dois anos. Todas as análises de regressão logística foram conduzidas tendo como variável dependente a ocorrência de diarréia no dia, informação disponível nos dois inquéritos.

\section{Impacto dos determinantes distais da doença diarréica}

O impacto potencial que variações entre 1984/ 85 e 1995/96 na renda familiar e na escolaridade materna exerceram sobre a prevalência da doença diarréica é examinado na Tabela 5. Os riscos rela- 
tivos de ocorrência de doença são estimados por um modelo de regressão logística que tem a ocorrência da doença diarréica no dia como variável dependente, a renda familiar per capita e a escolaridade materna como variáveis potencialmente explanatórias e a idade da criança e o ano do inquérito como variáveis de controle. $\mathrm{O}$ não controle no modelo quanto a determinantes pertencentes a níveis hierárquicos inferiores à renda familiar e à escolaridade materna, como o saneamento e o aleitamento materno, atende às especificações do modelo hierárquico e implica que essas variáveis poderão vir a ser os fatores através dos quais a renda e a escolaridade interferirão com o risco da doença diarréica.

Tanto a renda familiar (deflacionada e expressa em salários-mínimos de outubro de 1996) quanto a escolaridade materna apresentaram melhorias entre os inquéritos realizados: por exemplo, quase duplica a proporção de crianças de mães com oito ou mais anos de escolaridade e mais do que duplica a proporção de crianças de famílias com renda de pelo menos dois salários-mínimos per capita. No entanto, associação significativa independente é demonstrada entre renda familiar e ocorrência de diarréia, mas não entre escolaridade materna e diarréia. De per si, a melhoria na renda familiar observada entre 1984/85 e 1995/96 levaria a prevalência instantânea da diarréia a se reduzir em pouco mais de $20 \%$. Vale lembrar que, no período, a prevalência instantânea da doença diarréica infantil na cidade de São Paulo declinou em cerca de $50 \%$.

\section{Impacto de determinantes intermediários da doença diarréica}

\section{Acesso a serviços públicos de água e esgoto}

No período decorrido entre os inquéritos, o acesso ao abastecimento de água torna-se virtualmente universal na cidade, diminuem as situações onde a água da rede não chega ao interior das residências e expande-se substancialmente a cobertura da rede de esgoto. Por outro lado, controlando-se a idade da criança, o ano do inquérito, a renda familiar e a escolaridade materna, vê-se que há uma relação inversa, do tipo dose-resposta, entre acesso à rede de água e ocorrência da doença diarréica $(\mathrm{p}<0,10)$. Embora também se constate uma relação inversa entre acesso à rede de esgoto e diarréia, nesse caso a associação é de fraca intensidade e não significativa $(\mathrm{p}=0,53)$. Levando em conta os riscos de doença diarréica associados à não cobertura ou à cobertura parcial ("ponto fixo") da rede de água da cidade, calcula-se que a expansão desse serviço no período 1984/85-1995/96 levaria a prevalência da diarréia infantil a se reduzir em 9,0\% (Tabela 6).

Tabela 5 - Variações temporais na renda familiar e na escolaridade materna e estimativa de seu impacto sobre a prevalência instantânea da doença diarréica. Crianças entre zero e 59 meses de idade da cidade de São Paulo, SP. 1984/85 e 1995/96.

\begin{tabular}{|c|c|c|c|c|}
\hline \multirow[b]{2}{*}{ Variável explanatória } & \multicolumn{2}{|c|}{$\begin{array}{c}\text { Distribuição (\%) } \\
\text { em }\end{array}$} & \multirow{2}{*}{$\begin{array}{l}\text { Risco relativo de } \\
\text { doença diarréica* }\end{array}$} & \multirow{2}{*}{$\begin{array}{l}\text { Variação esperada } \\
\text { na prevalência de } \\
\text { doença diarréica** }\end{array}$} \\
\hline & $1984 / 85$ & $1995 / 96$ & & \\
\hline $\begin{array}{l}\text { Renda familiar per capita } \\
0,0,5 \\
0,5-2,0 \\
2,0 r+ \\
\text { Escolaridade materna (anos) }\end{array}$ & $\begin{array}{l}23,0 \\
62,5 \\
14,5\end{array}$ & $\begin{array}{r}9,3 \\
55,6 \\
35,1\end{array}$ & $\begin{array}{r}\mathrm{p}<0,05 \\
3,63 \\
1,20 \\
1,00 \\
\mathrm{p}=0,56\end{array}$ & $-21,6 \%$ \\
\hline $\begin{array}{l}0-3 \\
4-7 \\
8 \mathrm{e}+ \\
\end{array}$ & $\begin{array}{l}34,2 \\
38,6 \\
27,2 \\
\end{array}$ & $\begin{array}{l}14,6 \\
38,6 \\
46,8 \\
\end{array}$ & $\begin{array}{l}1,57 \\
1,06 \\
1,00 \\
\end{array}$ & - \\
\hline
\end{tabular}

*0 risco relativo de doença diarréica corresponde ao "odds ratio" ajustado para idade, ano do inquérito e escolaridade materna (ou, alternativamente, renda familiar per capita) fornecido por análise de regressão logística aplicada ao conjunto das crianças estudadas em 1984/85 e 1995/96.

**Resulta da comparação entre os resultados que se obtêm ao se ponderar os riscos relativos de doença diarréica segundo a distribuição da variável explanatória no primeiro e no segundo inquérito.

Tabela 6 - Variações temporais no acesso à rede pública de abastecimento de água e de coleta de esgoto e estimativa de seu impacto sobre a prevalência instantânea da doença diarréica. Crianças entre zero e 59 meses de idade da cidade de São Paulo, SP, $1984 / 85$ e 1995/96.

\begin{tabular}{|c|c|c|c|c|}
\hline \multirow[b]{2}{*}{ Variável explanatória } & \multicolumn{2}{|c|}{$\begin{array}{c}\text { Distribuição (\%) } \\
\text { em }\end{array}$} & \multirow{2}{*}{$\begin{array}{l}\text { Risco relativo de } \\
\text { doença diarréica* } \\
(\mathrm{n}=2.296)\end{array}$} & \multirow{2}{*}{$\begin{array}{c}\text { Variação esperada na } \\
\text { na prevalência de } \\
\text { doença diarréica }\end{array}$} \\
\hline & $\begin{array}{l}1984 / 85 \\
(n=1.016)\end{array}$ & $\begin{array}{c}1995 / 96 \\
(n=1.280)\end{array}$ & & \\
\hline Rede de água & & & $\mathrm{p}=0,09 * *$ & \\
\hline Canalizada & 87,7 & 94,4 & 1,00 & $-9,0 \%$ \\
\hline "Ponto fixo" & 7,6 & 5,3 & 2,07 & \\
\hline Ausente & 4,7 & 0,3 & 2,80 & \\
\hline \multicolumn{5}{|l|}{ Rede de esgoto } \\
\hline Presente & 44,1 & 71,6 & 1,00 & - \\
\hline Ausente & 55,9 & 28,4 & 1,29 & \\
\hline
\end{tabular}

*Calculado como na Tabela 5, acrescendo-se às variáveis de controle a renda familiar e a escolaridade materna.

**Tendência linear. 
Tabela 7 - Aleitamento materno: variações temporais e associação com a prevalência instantânea da doença diarréica. Crianças entre zero e 23 meses de idade da cidade de São Paulo, SP, 1984/85 e 1995/96.

\begin{tabular}{|c|c|c|c|}
\hline \multirow[b]{2}{*}{ Aleitamento materno } & \multicolumn{2}{|c|}{ Distribuição (\%) } & \multirow{2}{*}{$\begin{array}{l}\text { Risco relativo de } \\
\text { doença diarréica* }\end{array}$} \\
\hline & $1984 / 85$ & $1995 / 96$ & \\
\hline $\begin{array}{l}\text { Presente } \\
\text { Ausente }\end{array}$ & $\begin{array}{l}23,9 \\
76,1\end{array}$ & $\begin{array}{l}32,6 \\
67,4\end{array}$ & $\begin{array}{r}p=0,30 \\
1,00 \\
2,91\end{array}$ \\
\hline
\end{tabular}

*Calculado como na Tabela 5, acrescendo-se às variáveis de controle a renda familiar, a escolaridade materna e o acesso às redes de água e de esgoto.

\section{Prática de aleitamento em menores de dois anos de idade}

A Tabela 7 descreve a evolução da freqüência do aleitamento materno entre as crianças menores de dois anos de idade estudadas pelos inquéritos de 1984/85 e de 1995/96, bem como a associação existente, nos dois inquéritos tomados em conjunto, entre amamentação e risco de diarréia. Nota-se que houve um discreto aumento na freqüência de crianças amamentadas: $23,9 \%$ no primeiro inquérito e $32,6 \%$ no segundo inquérito. Vê-se também que a diarréia é quase três vezes mais freqüente em crianças não amamentadas, mesmo quando são controladas diversas variáveis de confusão (idade, ano do inquérito, renda familiar, escolaridade materna e acesso às redes de água e esgoto). Entretanto, possivelmente pela restrição das amostras aos menores de dois anos, a associação entre ocorrência de diarréia e amamentação não chegou a alcançar significado estatístico $(\mathrm{p}=0,30)$. Por essa razão, não se pôde estimar o possível impacto sobre a prevalência da diarréia decorrente do progresso observado na prática da amamentação (esse impacto, se calculado, equivaleria a uma redução de $6,8 \%$ na prevalência instantânea da doença diarréica).

\section{ISC U SSÃO}

$\mathrm{O}$ inquérito domiciliar mais recente realizado em São Paulo (1995/96) indica que a cidade desfruta de situação privilegiada quanto à frequiência da diarréia infantil. A estimativa de cerca de um episódio de diarréia por criança-ano aproxima São Paulo da situação encontrada, por exemplo, nos Estados Unidos 1,3 episódios por criança-ano ${ }^{7}$ - e distancia a cidade, em muito, da situação vista na maior parte dos países em desenvolvimento., ${ }^{4,12}$ Comparações com inquéritos realizados em outras regiões brasileiras são igualmente favoráveis à cidade de São Paulo. ${ }^{3,17} \mathrm{Na}$ área metropolitana de Recife, por exemplo, inquérito domiciliar realizado em 1997 encontrou 5,6\% de crianças com diarréia no dia da entrevista $(0,9 \%$ em São Paulo) e 16,9\% com diarréia nas duas últimas semanas $\left(4,7 \%\right.$ em São Paulo). ${ }^{10}$

Vale notar, entretanto, que freqüências de diarréia infantil não muito distantes daquelas observadas em
Recife foram encontradas entre famílias de São Paulo que vivem em extrema pobreza (menos de 0,5 salário-mínimo per capita): $2,4 \%$ de crianças com diarréia no dia da entrevista e 9,3\% nas duas últimas semanas. Essa situação indica a possibilidade (e a necessidade) de reduções adicionais na prevalência da diarréia infantil em São Paulo.

O declínio na frequêencia da doença diarréica na população infantil de São Paulo já havia sido documentado entre meados das décadas de 70 e $80 .{ }^{2} \mathrm{O}$ presente estudo ratifica essa tendência e indica que ela ocorreu de forma particularmente intensa no terço das crianças mais pobres da cidade. A escassez de inquéritos sequienciais e comparáveis sobre a prevalência da diarréia infantil em nosso meio torna difícil averiguar a existência de tendências semelhantes em outras cidades brasileiras. Na área metropolitana de Recife, inquéritos domiciliares realizados em 1991 e 1997 indicam ligeiro aumento na prevalência instantânea da diarréia infantil: $4,1 \%$ e $5,6 \%$, respectivamente. ${ }^{8,10}$

As razões subjacentes à redução da prevalência da diarréia infantil na cidade de São Paulo foram examinadas a partir de procedimentos analíticos que envolveram o estudo dos determinantes da ocorrência da doença na cidade e a evolução da freqüência desses determinantes no período 1984/851995/96. Em essência, demonstrou-se que mudanças positivas no poder aquisitivo das famílias e a expansão da cobertura do serviço público de abastecimento de água da cidade justificariam parte considerável do declínio observado na prevalência da diarréia infantil, havendo, ainda, evidência de que um discreto aumento na frequiência da amamentação, nos primeiros dois anos de vida, possa ter auxiliado a redução da enfermidade.

Por fim, cumpre notar que o declínio na incidência de hospitalizações por diarréia na cidade de São Paulo, mais intenso do que o declínio na prevalência da doença, pode estar refletindo, a exemplo do documentado em outras regiões do País, ${ }^{5,18}$ melhorias no manejo ambulatorial da enfermidade, incluindo o emprego mais freqüente da terapia de reidratação oral, cuja disseminação no país ocorre a partir da segunda metade da década de 80. 


\section{REFERÊNCIAS}

1. Bairagi R, Chowdhury MK, Kim YJ, Curlin GT, Gray RH The association between malnutrition and diarrhoea in rural Bangladesh. Int J Epidemiol 1987;16:477-81.

2. Benicio MHD'A, Monteiro CA, Pino ZHP, Rio EMB Estudo das condições de saúde das crianças do Município de São Paulo, SP 9 Brasil, 1984-1985. IV. D oença diarréica. Rev Saúde Pública 1987;21:23-8.

3. Benicio MHD'A, Cesar CLG, Gouveia NC. Perfil de morbidade e padrão de utilização de serviços de saúde das crianças brasileiras menores de cinco anos - 1989. In: Monteiro MFG, Cervini R, organizadores. Perfil estatístico de crianças e mães no Brasil. Aspectos de saúde e nutrição de crianças no Brasil 1989. Rio de Janeiro: IBGE/U NICEF; 1992. p. 79-96.

4. Bern C, Martines J, Zoysa I, G lass RI. The magnitude of the global problem of diarrhoeal disease: a ten-year update. Bull World Health O rgan 1992;70:705-14.

5. César JA, Victora CG, Barros FC, Ramos FA, Albernaz $E P$, O liveira LM, et al. Hospitalizações em menores de um ano pertencentes a duas coortes de base populacional no Sul do Brasil: tendências e diferenciais. Cad Saúde Pública 1996;12Supl 1:67-71.

6. Esrey AS, Feachem RG, Hughes JM. Interventions for the control of diarrhoeal diseases in young children: improving water supply and excreta disposal facilities. Bull World Health O rgan 1985;63:757-72.

7. Glass RI, Lew JF, Gangarosa RE, LeBaron CW, Ho MS. Estimates of the morbidity and mortality from diarrheal diseases in American children. J Pediatr 1991;118:527-33.

8. Governo do Estado de Pernambuco/U N ICEF. Crianças e adolescentes em Pernambuco: saúde, educação e trabalho. Recife: Governo do Estado de Pernambuco/ UNICEF; 1992.

9. Guerrant RL, Kirchhoff LV, Shields DS, Nations MK, Leslie J, Souza MA, et al. Prospective study of diarrhoeal illnesses in N ortheastern Brazil: patterns of disease, nutritional impact, etiologiy and risk factors. J Infect Dis 1983;148:986-97.

10. Instituto Nacional de Alimentação e N utrição. II Pesquisa Estadual de Saúde e Nutrição - 1997: saúde, nutrição, alimentação e condições socioeconômicas no Estado de Pernambuco. Recife: IN AN/M S-DN/U FPESES/PE; 1998.

11. Kirkwood BT. Essentials of medical statistics. London: Blackwelll; 1988.
12. M artines J, Phillips M, Feachem RG. Diarrheal diseases. The public health significance of diarrhea lowering disease incidence. In: Jamison DT, Mosley WH, M easham AR, Bobadilla JL. Disease control priorities in developing countries. Washington (DC): O xford Medical Publications; 1993. p. 91-116.

13. Monteiro CA, Pino ZHP, Benicio MHD'A, Szarfarc SC. Estudo das condições de saúde das crianças do Município de São Paulo (1984/85). I. Aspectos metodológicos, características sócio-econômicas e ambiente físico. Rev Saúde Pública 1986;20:435-45.

14. M onteiro CA. Saúde e nutrição das crianças de São Paulo: diagnóstico, contrastes sociais e tendências. São Paulo: Hucitec/EDU SP; 1988.

15. M onteiro CA, Nazário CL. Declínio da mortalidade infantil e eqüidade social: o caso da cidade de São Paulo entre 1973 e 1993. In: Monteiro CA, organizador. Velhos e novos males da saúde no Brasil: a evolução do país e de suas doenças. São Paulo: Hucitec/N U PENSUSP; 1995. p. 173-85.

16. M onteiro $C A$, Silva $N N$, N azário $C L$. A pesquisa de campo 1995/96. In: Monteiro CA, organizador. Como e por que melhoram (ou pioram) os indicadores de saúde e nutrição na infância? 0 caso da cidade de São Paulo na segunda metade do século XX. São Paulo: NU PENS/ USP; 1999. (Relatório Técnico - FAPESP, julho/1999).

17. Victora CG, Barros FC, H orta BL, Cesar JA. Saúde e nutrição das crianças nordestinas. Pesquisas estaduais 1987-1992. Brasília: UNICEF; 1995.

18. Victora CG, O linto MRA, Barros FC, N obre LC. Falling diarrhoea mortality in Northeastern Brazil: did ORT play a role? Health Pol Plan 1996;11:132-41.

19. Victora CG, Huttly SR, Fuchs SC, O linto MTA. The role of conceptual frameworks in epidemiological analysis: a hierarchical approach. Int J Epidemiol 1997;26:224-7.

20. Waldman EA, Silva LJ, Monteiro CA. Trajetória das doenças infecciosas: da eliminação da poliomielite à reintrodução da cólera. In: Monteiro CA, organizador. Velhos e novos males da saúde no Brasil: a evolução do país e de suas doenças. São Paulo: Hucitec/NU PENSUSP; 1995. p. 195-246.

21. World Health O rganization. (1994). Household survey manual: diarrhoea and acute respiratory infections. Geneva: WHO ; 1994. (WHO/CDR/94.8). 